\title{
MEASUREMENT OF THE SELF-BROADENING RATE COEFFICIENTS OF THE CESIUM RESONANCE LINES
}

\author{
Z. J. JABBOUR, $\dagger$ J. SAGLE, R. K. NAMIOTKA, and J. HUENNEKENS \\ Department of Physics, 16 Memorial Drive East, Lehigh University, Bethlehem, PA 18015, U.S.A.
}

(Received 10 February 1995)

\begin{abstract}
The self-broadening rate coefficients of the cesium $D_{1}\left[6^{2} S_{1 / 2}-6^{2} P_{1 / 2}\right]$ and $D_{2}$ $\left[6^{2} S_{1 / 2}-6^{2} P_{3 / 2}\right]$ resonance lines are determined from the $6^{2} P_{1 / 2} \rightarrow 6^{2} D_{3 / 2}, 6^{2} P_{3 / 2} \rightarrow 7^{2} D_{3 / 2}$ and $6^{2} P_{3,2} \rightarrow 7^{2} D_{5 / 2}$ transition lineshapes, which are mapped out using single-mode $\mathrm{cw}$ lasers. The self-broadening rates are found from Voigt function fits (including hyperfine structure) to the experimental lineshapes. Our measured $D_{1}$ and $D_{2}$ broadening coefficients, $k_{\mathrm{br}}\left(D_{1}\right)=$ $(5.7 \pm 1.0) \times 10^{-7} \mathrm{~cm}^{3} \mathrm{sec}^{-1}$ and $k_{\mathrm{br}}\left(D_{2}\right)=(6.7 \pm 1.1) \times 10^{-7} \mathrm{~cm}^{3} \mathrm{sec}^{-1}$, are compared to previous experimental and theoretical values in the literature. The present $D_{2}$ broadening coefficient is smaller, and the $D_{1}$ broadening coefficient is larger, than theoretical values.
\end{abstract}

\section{INTRODUCTION}

The study of pressure broadening of spectral lines provides information on fundamental atom-atom interactions and tests theoretical models of collisional broadening and calculated long-range potentials. Resonance broadening has been studied by measurements of absorption coefficients in the wings of resonance lines, ${ }^{18}$ and by observing transitions between resonance levels and more highly excited states. ${ }^{9-12}$ Recently, the cesium resonance broadening rates have been measured using Doppler-free selective reflection techniques, ${ }^{13,14}$ although there is some ambiguity as to how the linewidth measured using this technique relates to the full-width-half-maximum of the absorption lineshape function $k_{v}$. Huennekens and Gallagher measured the resonance broadening rate coefficients for sodium by spectral separation of Rayleigh and fluorescence light, coupled with an independent calibration of the ground state density based on measurements of absolute line-center and line-wing absorption coefficients. ${ }^{15}$ Their measurements agreed, to within the estimated 15\% experimental uncertainties, with calculations from the resonance broadening theories of Carrington, Stacey, and Cooper, ${ }^{16}$ Ali and Griem, ${ }^{17}$ and Reck et al ${ }^{18}$ Other predictions from these theoretical models, involving $J=0$ to $J=1$ transitions, have also been experimentally verified to within $5-10 \% .^{19}$

In our lab we have observed a systematic discrepancy, in the case of cesium in sealed vacuum cells, between densities obtained from vapor pressure formulas and equivalent-width measurements based upon the theoretical broadening rates of Carrington, Stacey, and Cooper. ${ }^{16}$ (These theoretical broadening rates were found to be reliable in the similar case of sodium.) This discrepancy, which could be as much as $50 \%$ in the temperature range $25-200^{\circ} \mathrm{C}$, implies either that the theoretical broadening rates are wrong, that the vapor pressure formulas are not accurate in this temperature range, or that the temperature of the liquid metal reservoir was not being measured accurately with a single thermocouple attached to the outside of the cell wall. Previous experiments measuring the resonance broadening rate coefficients in cesium were performed in absorption ${ }^{4-7}$ or in selective reflection ${ }^{13,14}$ and they all relied on density measurements from vapor pressure formulas. Some of these measurements yield results which also disagree with the theoretical broadening rates.

One complication in the interpretation of this situation is that different types of measurements probe different regions of the line. In the far wings of the line, i.e., for $\Delta \omega \gg 1 / \tau_{c}$ where $\tau_{c}$ is the 
collision duration, the quasi-static theory is valid. The line core region is described by the impact approximation which is valid for detunings which satisfy $\Delta \omega \ll 1 / \tau_{\mathrm{c}}$. Thus the two regimes are formally divided by the Weisskopf frequency $\Delta \omega_{\mathrm{c}} \sim 1 / \tau_{\mathrm{c}}$ which is $3.5-4.5 \times 10^{10} \mathrm{sec}^{-1}$ for self-broadening of the cesium resonance lines. ${ }^{20}$ Previous absorption measurements ${ }^{4-7}$ have provided information on the line wings. Such experiments actually measure an effective $C_{3}$ coefficient which can be formally related to the line core broadening parameter $k_{\mathrm{br}}$. However, discrepancies between broadening rates measured in the line wings and line core certainly can exist. In addition, the large hyperfine structure of cesium (particularly the ground $F=3-F=4$ level splitting of $9.192 \mathrm{GHz}$ ) ${ }^{21}$ can contribute to the apparent broadening of the line. To the best of our knowledge, only the selective reflection measurements of resonance broadening in ccsium havc taken hypcrfinc structurc into account, since the individual hyperfine components are resolved in that case. ${ }^{13,14}$

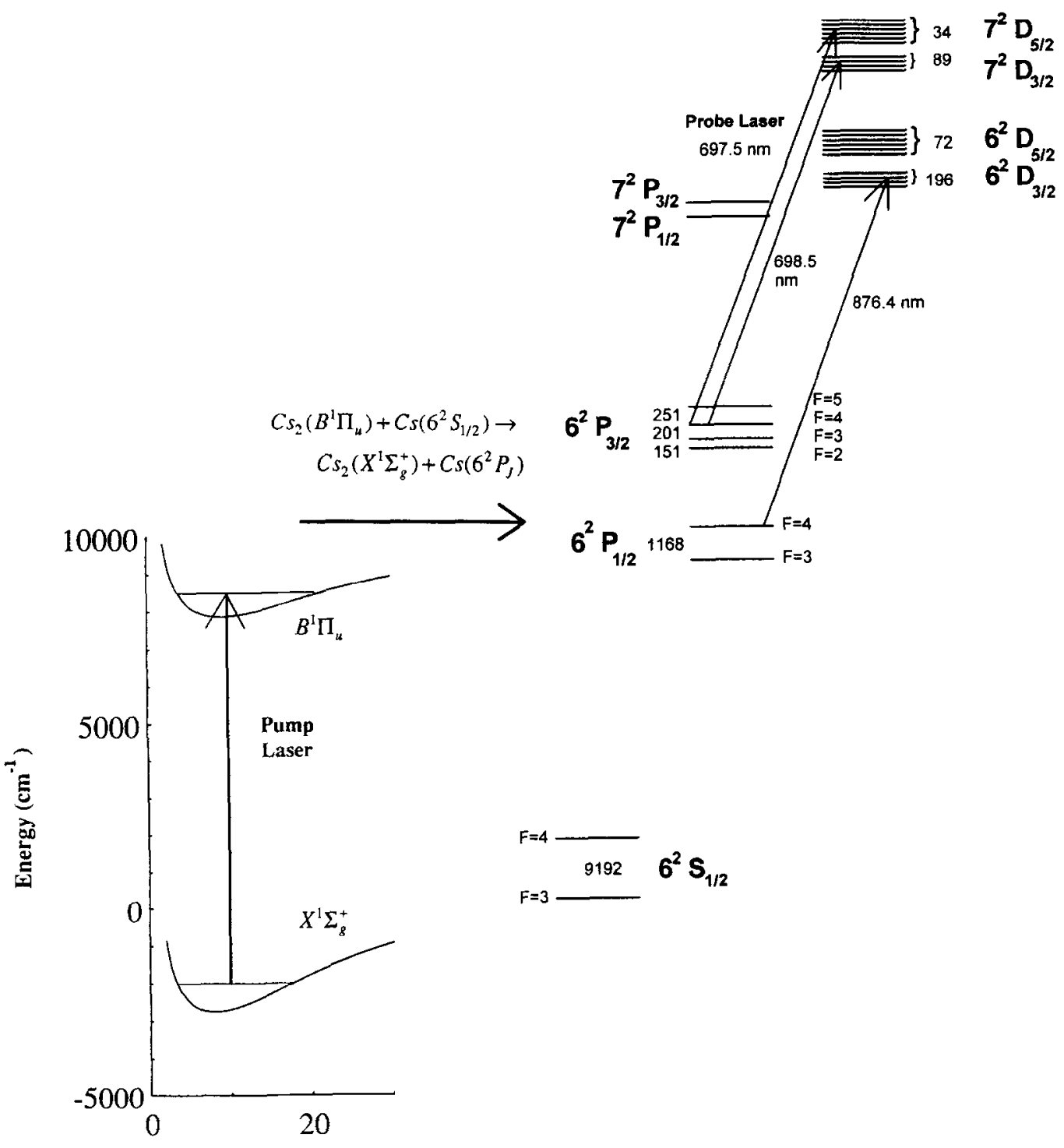

Internuclear Separation (A)

Fig. 1. Schematic energy level diagram of cesium showing the purnping scheme used in the present resonance broadening studies. The pump laser excites $\mathrm{Cs}_{2}$ molecules on the $B^{\prime} \mathrm{II}_{u} \leftarrow X^{\prime} \Sigma_{\mathrm{g}}^{+}$transition. Population is then transferred to the $6^{2} P_{J}$ levels through the collisional process $\mathrm{Cs}_{2}\left(B^{1} \mathrm{II}_{\mathrm{u}}\right)+\operatorname{Cs}\left(6^{2} S_{1 / 2}\right) \rightarrow \mathrm{Cs}_{2}\left(X^{1} \Sigma_{\mathrm{g}}^{+}\right)+\operatorname{Cs}\left(6^{2} P_{J}\right)$. The $6^{2} P_{J}$ lineshapes are probed on the transitions indicated. The $6^{2} P_{3 / 2} \rightarrow 7^{2} D_{3 / 25 / 2}$ absorption lineshapes are recorded by scanning the probe laser across those transitions while monitoring fluorescence on the $7^{2} D_{3 / 2} \rightarrow 6^{2} P_{1 / 2}$ transition at $672.5 \mathrm{~nm}$. The $6^{2} P_{1 / 2} \rightarrow 6^{2} D_{3 / 3}$ absorption lineshape is recorded by scanning the probe laser over that line while monitoring

$7^{2} P_{3 / 2} \rightarrow 6^{2} S_{1 / 2}$ cascade fluorescence at $455 \mathrm{~nm}$. Hyperfine structure splittings are given in $\mathrm{MHz}$. 
For these reasons we decided to measure the impact-regime cesium resonance broadening rates under conditions where the cell temperature could be accurately monitored at a number of positions along the cell body. In addition, the method of analysis would allow the full hyperfine structure to be taken into account.

In this experiment, we measured the broadening rates of transitions between the resonance levels and more highly excited states. A diagram showing the relevant energy levels of cesium is given in Fig. 1. A single-mode $\mathrm{cw}$ laser operating at 755 or $816 \mathrm{~nm}$ pumped $\mathrm{Cs}_{2}$ molecules into excited ro-vibrational levels of the $B^{1} \mathrm{II}_{\mathrm{u}}$ state and collisions transferred population to the cesium atomic $6^{2} P_{1 / 2}$ and $6^{2} P_{3 / 2}$ states. A second tunable, single-mode, cw laser was then scanned across either the $6^{2} P_{1 / 2} \rightarrow 6^{2} D_{3 / 2}$ or $6^{2} P_{3 / 2} \rightarrow 7^{2} D_{J}$ transition while the $7^{2} P_{3 / 2} \rightarrow 6^{2} S_{1 / 2}$ or $7^{2} D_{3 / 2} \rightarrow 6^{2} P_{1 / 2}$ fluorescence was monitored, respectively. In this manner, we mapped out the $6^{2} P_{1 / 2} \rightarrow 6^{2} D_{3 / 2}$ and $6^{2} P_{3 / 2} \rightarrow 7^{2} D_{J}$ transition lineshapes for different cell temperatures. The broadening rates were obtained from Voigt function fits to these experimental lineshapes.

In this analysis, we have assumed that the resonance broadening rate coefficient of the $6^{2} P_{1 / 2} \rightarrow 6^{2} D_{3 / 2}\left(6^{2} P_{3 / 2} \rightarrow 7^{2} D_{J}\right)$ transition is the same as that for the $6^{2} S_{1 / 2} \rightarrow 6^{2} P_{1 / 2}\left(6^{2} S_{1 / 2} \rightarrow 6^{2} P_{3 / 2}\right)$ transition due to the nature of the long range interaction potentials involved. The resonance interaction between a ground state $\left(6^{2} S_{1 / 2}\right)$ cesium atom and one in the first excited state $\left(6^{2} P_{J}\right)$ can be described by the molecular potentials at large internuclear separations $R$, which obey: ${ }^{22}$

$$
\Delta V=V(R)-V(\infty)=\hbar C_{3} / R^{3},
$$

while the van der Waals $\left(6^{2} S_{1 / 2}+6^{2} S_{1 / 2}\right)$, and the resonance quadrupole-quadrupole $\left(n^{2} D_{J}+6^{2} S_{1 / 2}\right)$ interactions can be described by $\hbar C_{6} / R^{6}$ and $\hbar C_{5} / R^{5}$, respectively. ${ }^{23}$ For a typical resonance broadening full-width-at-half-maximum, $\Gamma_{\mathrm{br}} \sim 2 \times 10^{10} \mathrm{sec}^{-1}$, and $C_{3} \sim 4 \times 10^{-8} \mathrm{~cm}^{3} \mathrm{sec}^{-1}$, ${ }^{16-18}$ we find that the pressure broadened lineshapes are dominated by the potentials at $R>100 \AA$. For this range, we find that $C_{3} / R^{3} \gg C_{5} / R^{5}, C_{6} / R^{6}$ where we have estimated $C_{5}$ and $C_{6}$ constants from values calculated for the analogous $\mathrm{Li}$ and $\mathrm{Na}$ systems by Bussery and Aubert-Frécon ${ }^{23}$ Therefore, ground state atom broadening of any transition involving the resonance $6^{2} P$, levels will be dominated by the resonance interaction potentials. Hence, the resonance broadening rate coefficients measured for $6^{2} P_{J} \rightarrow n^{2} D_{J}$ transitions are, effectively, equal to that for the $6^{2} S_{1 / 2} \rightarrow 6^{2} P_{J}$ transition.

\section{EXPERIMENT}

A standard pump-probe experimental setup was used in this work. A cylindrical Pyrex glass cell, $7.65 \mathrm{~cm}$ long and $2.20 \mathrm{~cm}$ in diameter, containing pure cesium (no buffer gas) was placed in a brass oven with quartz windows and heated with heating tape. The temperature was monitored by three thermocouples placed at various positions around the cell. The lowest measured temperature point was varied in the range $272-326^{\circ} \mathrm{C}$ and it was verified that droplets of liquid metal sat at this lowest temperature position.

For cesium $D_{2}\left(6^{2} S_{1 / 2}-6^{2} P_{3 / 2}\right)$ line-broadening studies, cesium molecules were excited on the $B^{\prime} I_{u} \leftarrow X^{\prime} \Sigma_{g}^{+}$transition at $816 \mathrm{~nm}$ using a tunable single-mode $\mathrm{cw}$ Ti:Sapphire laser (Coherent Autoscan 899-29), pumped by $10 \mathrm{~W}$ from an argon ion laser. Typical Ti: Sapphire laser power was $760 \mathrm{~mW}$. Collisional excitation transfer from molecules to atoms then populated the $6^{2} P_{J}$ levels: $\mathrm{Cs}_{2}\left(B^{1} \mathrm{II}_{u}\right)+\operatorname{Cs}\left(6^{2} S_{\mathrm{J} / 2}\right) \rightarrow \mathrm{Cs}_{2}\left(X^{1} \Sigma_{\mathrm{g}}^{+}\right)+\operatorname{Cs}\left(6^{2} P_{J}\right)$. The $6^{2} P_{3 / 2} \rightarrow 7^{2} D_{3 / 2,5 / 2}$ atomic transitions at wavelengths of 698.5 and $697.5 \mathrm{~nm}$, respectively, were probed using a tunable, single-mode $\mathrm{cw}$ ring dye laser (Coherent Autoscan 699-29), operating with LD700 dye (Rhodamine 700) and pumped by a 4-6 W krypton-ion laser. The dye laser produced $\sim 200 \mathrm{~mW}$ at these wavelengths, but this power was attenuated to less than $1 \mathrm{~mW}$ to avoid optical hyperfine pumping in the $6^{2} P_{3 / 2}$ state. The pump and probe laser beams were both gently focused into the cell. They counterpropagated through the vapor and were spatially overlapped with 2.0 and $1.5 \mathrm{~mm}$ beam diameters, respectively. The beam diameters did not vary significantly over the length of the cell.

For $D_{1}\left(6^{2} S_{1 / 2}-6^{2} P_{1 / 2}\right)$ studies, the roles of the dye and $\mathrm{Ti}$ :Sapphire lasers were reversed. The dye laser, with power of $\sim 100 \mathrm{~mW}$, was tuned to $755.5 \mathrm{~nm}$ to pump the $\mathrm{Cs}_{2}\left(B^{1} I_{u}\right)$ state. The Ti:Sapphire laser, with power attenuated down to $\sim 1 \mathrm{~mW}$ was scanned across the $6^{2} P_{1 / 2} \rightarrow 6^{2} D_{3 / 2}$ probe transition at $876.4 \mathrm{~nm}$. In this case, neither laser was focused, but the beams were apertured to diameters similar to those used in the $D_{2}$ studies.

In both cases, fluorescence was detected at right angles to the propagation direction of the lasers. 
For $D_{2}$ studies, the ring dye laser was scanned across the $6^{2} P_{3 / 2} \rightarrow 7^{2} D_{3 / 25 / 2}$ transitions while the $7^{2} D_{3 / 2} \rightarrow 6^{2} P_{1 / 2}$ transition fluorescence at $672.5 \mathrm{~nm}$ was imaged onto the entrance slit of a $0.22 \mathrm{~m}$ monochromator (Spex model 1681) and detected with a photomultiplier tube (PMT). The monochromator slits were opened to $1000 \mu \mathrm{m}$ (3000 $\mu \mathrm{m}$ when signals were weak) yielding a resolution of $\sim 3.6 \mathrm{~nm}(\sim 10.8 \mathrm{~nm})$, which was sufficient to resolve the fine-structure transitions. For $D_{1}$ measurements, the Ti:Sapphire laser was scanned across the $6^{2} P_{1 / 2} \rightarrow 6^{2} D_{3 / 2}$ transition while the monochromator was set to pass the $7^{2} P_{3 / 2} \rightarrow 6^{2} S_{1 / 2}$ cascade fluorescence at $455 \mathrm{~nm}$. In each case, the probe laser was chopped, and the PMT signals were processed with a lock-in amplifier and sent to one of the input channels of the Autoscan laser data acquisition system. Fluorescence was detected while scanning the probe laser for different cell temperatures ranging from 272 to $326^{\circ} \mathrm{C}$. Total $6^{2} P_{1 / 2} \rightarrow 6^{2} S_{1 / 2}$ or $6^{2} P_{3 / 2} \rightarrow 6^{2} S_{1 / 2}$ fluorescence was detected using a free-standing PMT with interference filter to insure against pump laser drift. Transmission of the probe laser intensity was also monitored to ensure that the probe beam was not being depleted as it passed through the cell. Tests were also carried out to verify that the pump and probe lasers, either individually or in combination, did not produce significant ionization (detected through the observation of fluorescence from high-lying atomic levels populated by recombination). It was also determined that no significant population of the $6^{2} D_{J}$ and $7^{2} P_{J}$ levels occurred through energy pooling processes, ${ }^{24}$ and that the probe laser by itself did not significantly populate the $6^{2} P$, levels. For each cell temperature, one or more probe laser scans were recorded. The dependence of the excitedstate-excited-state transition lineshape on probe laser power was studied to verify that there was no lineshape distortion due to optical hyperfine pumping within the $6^{2} P_{J}$ state.

\section{RESULTS AND DISCUSSION}

Typical experimental $6^{2} P_{1 / 2} \rightarrow 6^{2} D_{3 / 2}, 6^{2} P_{3 / 2} \rightarrow 7^{2} D_{3 / 2}$, and $6^{2} P_{3 / 2} \rightarrow 7^{2} D_{5 / 2}$ transition lineshapes, obtained at different cell temperatures, are shown in Figs. 2, 3, and 4. In general, these lineshapes

$$
\text { Cesium } 6 \mathrm{P}_{1 / 2} \rightarrow 6 \mathrm{D}_{3 / 2}
$$

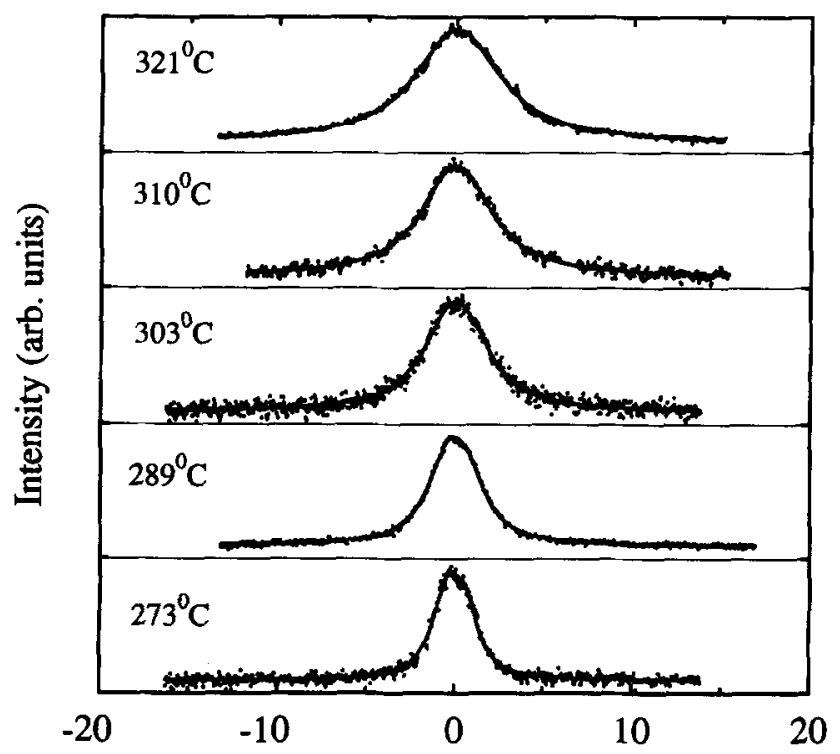

$\Delta \mathrm{v}(\mathrm{GHz})$

Fig. 2. Experimental lineshapes for the cesium $6^{2} P_{1 / 2} \rightarrow 6^{2} D_{3 / 2}$ transition as a function of temperature. The lower $6^{2} P_{1 / 2}$ level is populated by collisions following pumping of the cesium dimer $B^{1} \mathrm{II}_{4}$ state with a dye laser tuned to $755 \mathrm{~nm}$. The $6^{2} P_{1 / 2} \rightarrow 6^{2} D_{3 / 2}$ transition lineshape is then mapped by scanning a single-mode Ti:Sapphire laser over that transition, while recording total fluorescence emitted on the $7^{2} P_{3 / 2} \rightarrow 6^{2} S_{1 / 2}$ transition at $455 \mathrm{~nm}$. The experimental lineshapes are fitted with a sum of 6 Voigt functions (see text) and these fitted curves are also plotted in the figure. Vertical scales are not the same for data recorded at different temperatures. 
Cesium $6 \mathrm{P}_{3 / 2} \rightarrow 7 \mathrm{D}_{3 / 2}$

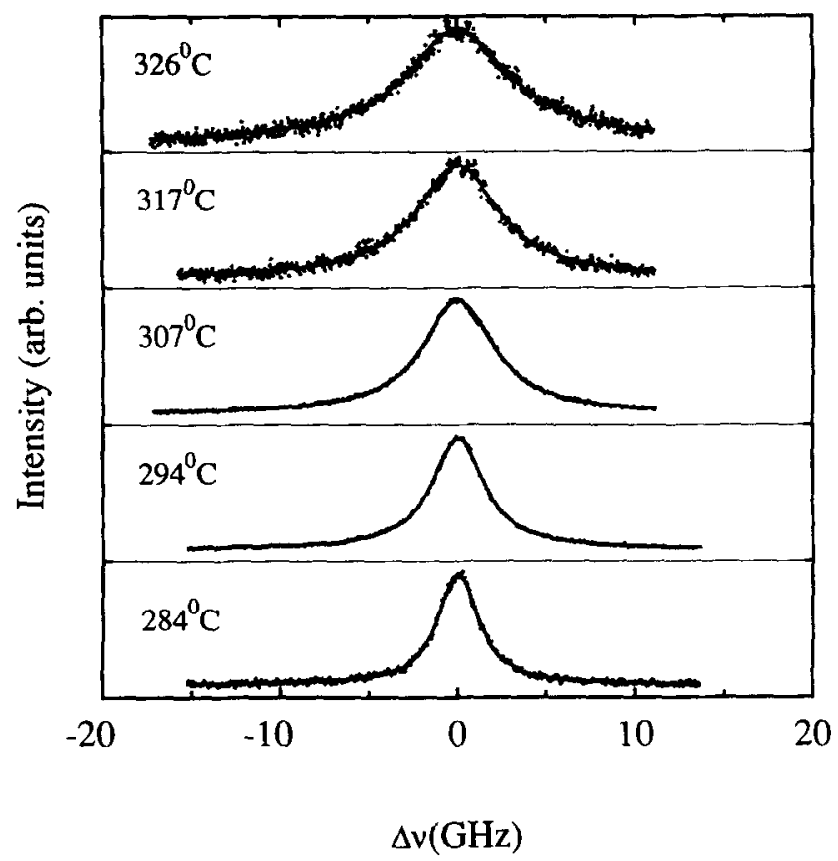

Fig. 3. Experimental lineshapes for the cesium $6^{2} P_{3 / 2} \rightarrow 7^{2} D_{3 / 2}$ transition as a function of temperature. The lower $6^{2} P_{3 / 2}$ level is populated by collisions following pumping of the cesium dimer $B^{1} I_{u}$ state with a $\mathrm{Ti}$ : Sapphire laser tuned to $816 \mathrm{~nm}$. The $6^{2} P_{3 / 2} \rightarrow 7^{2} D_{3 / 2}$ transition lineshape is then mapped by scanning a single-mode dye laser over that transition, while recording total fluorescence emitted on the $7^{2} D_{3 / 2} \rightarrow 6^{2} P_{1 / 2}$ transition at $672.5 \mathrm{~nm}$. The experimental lineshapes are fitted with a sum of 10 Voigt functions (see text) and these fitted curves are also plotted in the figure. Vertical scales are not the same for data recorded at different temperatures.

are affected by natural, Doppler, and pressure broadening, as well as by hyperfine structure. Hyperfine structure of the $6^{2} P_{1 / 2}$ state $\left({ }^{133} \mathrm{Cs}\right.$ has a nuclear spin $\left.I=7 / 2\right)$ results in two components, corresponding to $F=3$ and 4 , which are split by $1.168 \mathrm{GHz}^{21}$ (see Fig. 1). The $6^{2} P_{3 / 2}$ state splits into four components corresponding to $F=2,3,4,5$; the splitting between the $F=2$ and $F=5$ levels being $604 \mathrm{MHz} .^{21}$ The $6^{2} D_{3 / 2}$ and $7^{2} D_{3 / 2}$ states each have four hyperfine levels ranging from $F=2$ to $F=5$ with total splittings of 196 and $89 \mathrm{MHz}$, respectively, while the $7^{2} D_{5 / 2}$ state has six hyperfine levels ranging from $F=1$ to $F=6$ with a total splitting of $34 \mathrm{MHz} .^{21}$ Allowed transitions between the two hyperfine manifolds of the studied transitions obey the selection rules $\Delta F=0, \pm 1$, and $\Delta m_{\mathrm{F}}=0$ for linearly polarized light. Therefore, the $6^{2} P_{1 / 2} \rightarrow 6^{2} D_{3 / 2}, 6^{2} P_{3 / 2} \rightarrow 7^{2} D_{3 / 2}$, and $6^{2} P_{3 / 2} \rightarrow 7^{2} D_{5 / 2}$ lineshapes are convolutions of six, ten, and twelve allowed transitions, respectively.

The Voigt function is a numerical convolution of a Gaussian (due to the Doppler effect) and a Lorentzian (due to natural and pressure broadening). It is characterized by the Voigt parameter ${ }^{25}$

$$
a=\frac{\sqrt{\ln (2)} \Gamma}{2 \pi \Delta v_{\mathrm{D}}},
$$

which is a measure of the ratio of the Lorentzian to Gaussian contributions to the lineshape. $\Delta v_{D}$ is the Doppler width (in $\mathrm{Hz}$ ) and is given by:

$$
\Delta v_{\mathrm{D}}=\frac{2}{\lambda_{0}}\left(\frac{2 R T(\ln 2)}{M}\right)^{1 / 2}
$$

and $\Gamma$ is the Lorentzian full-width-half-maximum in angular frequency units and is given by:

$$
\Gamma=\Gamma_{\text {nat }}+\Gamma_{\mathrm{br}}=\Gamma_{\text {nat }}+k_{\mathrm{br}} n .
$$


Cesium $6 \mathrm{P}_{3 / 2} \rightarrow 7 \mathrm{D}_{5 / 2}$

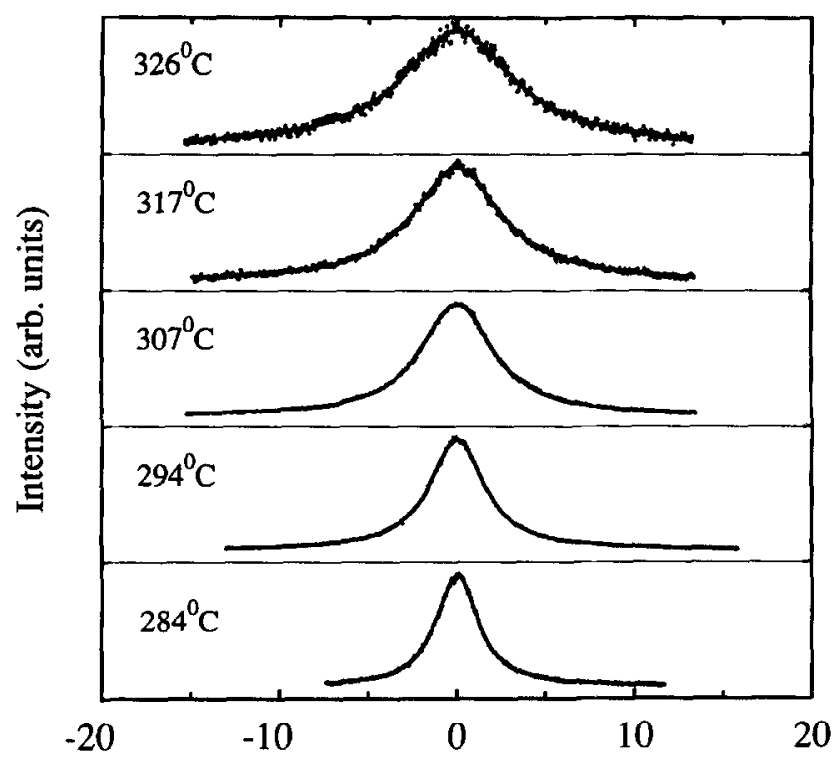

$\Delta v(\mathrm{GHz})$

Fig. 4. Experimental lineshapes for the cesium $6^{2} P_{3 / 2} \rightarrow 7^{2} D_{5 / 2}$ transition as a function of temperature. The lower $6^{2} P_{3 / 2}$ level is populated by collisions following pumping of the cesium dimer $B^{1} I_{u}$ state with a Ti:Sapphire laser tuned to $816 \mathrm{~nm}$. The $6^{2} P_{3 / 2} \rightarrow 7^{2} D_{5 / 2}$ transition lineshape is then mapped by scanning a single-mode dye laser over that transition, while recording total fluorescence emitted on the $7^{2} D_{3 / 2} \rightarrow 6^{2} P_{1 / 2}$ transition at $672.5 \mathrm{~nm}$. The experimental lineshapes are fitted with a sum of 12 Voigt functions (see text) and these fitted curves are also plotted in the figure. Vertical scales are not the same for data recorded at different temperatures.

In these equations, $\lambda_{0}$ is the transition wavelength, $R$ is the gas constant, $T$ is the temperature, $M$ is the cesium molar mass, $\Gamma_{\text {nat }}$ is the spontaneous emission rate (Einstein $A$ coefficient) of the transition, $k_{\mathrm{br}}$ is the self-broadening rate coefficient, and $n$ represents the cesium atom density.

We fit the experimental lineshapes with a sum of either six, ten, or twelve Voigt functions (one for each hyperfine component). Each Voigt is weighted by the square of the dipole matrix element of the hyperfine transition, and it is assumed that the lower $\sigma^{2} P_{J}$ hyperfine levels are collisionally populated in their statistical ratios. Because $\Gamma_{\text {nat }}$ of the transitions and the hyperfine splittings of the $6^{2} P_{J}$ states are known from Refs. 26 and 21 , respectively, and the Doppler width only depends on temperature [see Eq. (2)], only the Lorentzian width $\Gamma$ (which is approximately equal to $\Gamma_{\mathrm{br}}$ since $\left.\Gamma_{b r} \gg \Gamma_{\text {nat }}\right)$ is a free parameter in each fit. From the best fit to the data, the broadening rate $\Gamma_{b r}$ was found for each transition at each temperature. These measured values, which lie in the range $1-5 \times 10^{10} \mathrm{sec}^{-1}$, are reported in Table 1 . We report these as impact regime values, although they are only marginally smaller than the Weisskopf frequency. The best fits, along with the experimental lineshapes, are plotted in Figs. 2, 3, and 4.

As can be seen from Eq. (3), determination of the self-broadening rate coefficients depends upon reliable knowledge of the cesium density. Determinations of atomic cesium densities generally rely on vapor pressure formulas such as Taylor-Langmuir' ${ }^{27}$ or Nesmeyanov's. ${ }^{28}$ The latter reference is a compilation of vapor pressure data from many sources (including Taylor and Langmuir). The use of any vapor pressure formula strictly depends on measuring the temperature of the liquid metal bath in the cell, which in equilibrium is usually the cell's coldest point. However, it should be noted that the Taylor-Langmuir data only covered the temperature range -35 to $73^{\circ} \mathrm{C}$, and that most of the data sets used to construct the various curves given in Refs. 27-32 do not cover the temperature range of the present experiment. Values of densities determined from these various vapor pressure formulas agree in our temperature range to only $30 \%$ or so (these values are given 
Table 1. Cesium $D_{1}$ and $D_{2}$ line resonance broadening rates measured in this work.

\begin{tabular}{|c|c|c|c|c|}
\hline \multicolumn{5}{|c|}{$\begin{array}{l}D_{1} \text { Line (studied through analysis of the } 6^{2} P_{12} \rightarrow 6^{2} D_{3 n} \text { probe transition } \\
\text { lineshape) }\end{array}$} \\
\hline Temperature $\left({ }^{\circ} \mathrm{C}\right)$ & $\begin{array}{l}\text { Cesium Density } \\
\left(\text { ( 10 10 }^{16} \mathrm{~cm}^{-3} \text { ) }\right. \\
\text { (Nesmeyanov) }\end{array}$ & $\begin{array}{l}\text { Cesium Density } \\
\left(\mathrm{x} 10^{16} \mathrm{~cm}^{-3}\right) \\
\text { (Other) }\end{array}$ & $\Gamma_{b r}\left(\times 10^{9} \mathrm{~s}^{-1}\right) \mathrm{r}$ & $\begin{array}{c}k_{b r}\left(x^{10^{-7}} \mathrm{~cm}^{3} \mathbf{s}^{-1}\right) \mathrm{g} \\
=\Gamma_{b r} / \mathbf{n}\end{array}$ \\
\hline 273 & 1.85 & $\begin{array}{l}1.60^{\mathrm{a}} \\
1.43^{\mathrm{b}} \\
1.82^{\mathrm{c}} \\
1.98^{\mathrm{d}} \\
1.84^{\mathrm{c}} \\
\end{array}$ & $\begin{array}{l}11.1 \pm 2.0 \\
11.1 \pm 2.0 \\
10.9 \pm 2.0\end{array}$ & $\begin{array}{l}6.00 \pm 1.08 \\
6.00 \pm 1.08 \\
5.89 \pm 1.08\end{array}$ \\
\hline 279 & 2.18 & & $\begin{array}{l}12.2 \pm 1.5 \\
11.8 \pm 1.5 \\
12.7 \pm 1.5\end{array}$ & \begin{tabular}{|l|}
$5.60 \pm 0.69$ \\
$5.41 \pm 0.69$ \\
$5.83 \pm 0.69$
\end{tabular} \\
\hline 289 & 2.83 & & $\begin{array}{l}16.9 \pm 1.0 \\
17.1 \pm 1.5\end{array}$ & $\begin{array}{l}5.97 \pm 0.35 \\
6.04 \pm 0.53\end{array}$ \\
\hline 297 & 3.47 & & $\begin{array}{l}18.3 \pm 2.0 \\
17.4 \pm 2.0 \\
18.9 \pm 2.0\end{array}$ & \begin{tabular}{|l|}
$5.27 \pm 0.58$ \\
$5.01 \pm 0.58$ \\
$5.45 \pm 0.58$
\end{tabular} \\
\hline 303 & 4.02 & & $\begin{array}{l}21.7 \pm 3.0 \\
22.0 \pm 3.0 \\
\end{array}$ & $\begin{array}{l}5.40 \pm 0.75 \\
5.47 \pm 0.75\end{array}$ \\
\hline 304 & 4.12 & & $22.4 \pm 3.0$ & $5 . \overline{44 \pm 0.73}$ \\
\hline 310 & 4.75 & & $\begin{array}{l}27.4 \pm 3.0 \\
27.2 \pm 3.0 \\
26.4 \pm 3.0 \\
\end{array}$ & $\begin{array}{l}5.77 \pm 0.63 \\
5.73 \pm 0.63 \\
5.56 \pm 0.63\end{array}$ \\
\hline 321 & 6.14 & $\begin{array}{l}5.21^{\mathrm{a}} \\
4.84^{\mathrm{b}} \\
6.00^{\mathrm{c}} \\
6.21^{\mathrm{d}} \\
6.03^{\mathrm{e}} \\
\end{array}$ & $\begin{array}{l}36.9 \pm 2.0 \\
34.6 \pm 2.0\end{array}$ & $\begin{array}{l}6.01 \pm 0.33 \\
5.64 \pm 0.33\end{array}$ \\
\hline
\end{tabular}

$D_{2}$ Line (studied through analysis of the $6^{2} P_{3 / 2} \rightarrow 7^{2} D_{3 n}$ probe transition lineshape)

\begin{tabular}{|c|c|c|c|c|}
\hline Temperature $\left({ }^{\circ} \mathrm{C}\right)$ & $\begin{array}{l}\text { Cesium Density } \\
\left(\times 10^{16} \mathrm{~cm}^{-3}\right) \\
(\text { Nesmeyanov) }\end{array}$ & $\begin{array}{l}\text { Cesium Density } \\
\left(\mathbf{x} 10^{16} \mathrm{~cm}^{-3}\right) \\
\text { (Other) }\end{array}$ & $\Gamma_{b r}\left(\times 10^{y} \mathrm{~s}^{-1}\right)^{q}$ & $\begin{array}{l}k_{h r}\left(\times 10^{-7} \mathrm{~cm}^{3} \mathrm{~s}^{-1}\right)^{\mathrm{g}} \\
\quad=\Gamma_{h r} / \mathrm{n}\end{array}$ \\
\hline 272.5 & 1.83 & $\begin{array}{l}1.58^{\mathrm{a}} \\
1.41^{\mathrm{b}} \\
1.79^{\mathrm{c}} \\
1.95^{\mathrm{d}} \\
1.81^{\mathrm{e}} \\
\end{array}$ & $11.7 \pm 1.0$ & $6.39 \pm 0.55$ \\
\hline 284 & 2.49 & & $16.3 \pm 1.0$ & $6.55 \pm 0.40$ \\
\hline 294.5 & 3.26 & & $21.3 \pm 0.5$ & $6.53 \pm 0.15$ \\
\hline 299 & 3.69 & & $\begin{array}{l}25.1 \pm 2.0 \\
27.5 \pm 4.0 \\
25.6 \pm 2.0 \\
27.9 \pm 2.0\end{array}$ & $\begin{array}{l}6.80 \pm 0.54 \\
7.45 \pm 1.08 \\
6.94 \pm 0.54 \\
7.56 \pm 0.54 \\
\end{array}$ \\
\hline 307 & 4.43 & & $30.0 \pm 0.8$ & $6.77 \pm 0.18$ \\
\hline 317 & 5.60 & & $\begin{array}{l}34.5 \pm 2.5 \\
33.8 \pm 2.5\end{array}$ & $\begin{array}{l}6.16 \pm 0.45 \\
6.04 \pm 0.45 \\
\end{array}$ \\
\hline 326 & 6.87 & $\begin{array}{l}5.82^{\mathrm{a}} \\
5.43^{\mathrm{b}} \\
6.72^{\mathrm{c}} \\
6.92^{\mathrm{d}} \\
6.74^{\mathrm{e}} \\
\end{array}$ & $45.7 \pm 3.0$ & $6.65 \pm 0.44$ \\
\hline
\end{tabular}




\begin{tabular}{|c|c|c|c|c|}
\hline \multicolumn{5}{|c|}{$\begin{array}{l}D_{2} \text { Line (studied through analysis of the } 6^{2} P_{3 / 2} \rightarrow 7^{2} D_{5 / 2} \text { probe transition } \\
\text { lineshape) }\end{array}$} \\
\hline Temperature $\left({ }^{\circ} \mathrm{C}\right)$ & $\begin{array}{l}\text { Cesium Density } \\
\left(\text { (x 10 }^{16} \mathrm{~cm}^{-3}\right) \\
\text { (Nesmeyanov) }^{28}\end{array}$ & $\begin{array}{l}\text { Cesium Density } \\
\left(x 1^{16} \mathrm{~cm}^{-3}\right) \\
\text { (Other) }\end{array}$ & $\Gamma_{h r}\left(\times 10^{\theta} \mathrm{s}^{-1}\right)^{p}$ & $\begin{array}{c}k_{h r}\left(\times 10^{-7} \mathrm{~cm}^{3} \mathrm{~s}^{-1}\right) \\
=\Gamma_{h r} / \mathrm{n}\end{array}$ \\
\hline 272.5 & 1.83 & $\begin{array}{l}1.58^{\mathrm{a}} \\
1.41^{\mathrm{b}} \\
1.79^{\mathrm{c}} \\
1.95^{\mathrm{d}} \\
1.81^{\mathrm{e}} \\
\end{array}$ & $12.4 \pm 1.0$ & $6.78 \pm 0.55$ \\
\hline 284 & 2.49 & & $\begin{array}{l}16.4 \pm 0.5 \\
17.6 \pm 0.5 \\
17.6 \pm 0.7 \\
17.7 \pm 0.7\end{array}$ & $\begin{array}{l}6.59 \pm 0.20 \\
7.07 \pm 0.20 \\
7.07 \pm 0.28 \\
7.11 \pm 0.28\end{array}$ \\
\hline 294.5 & 3.26 & & \begin{tabular}{|l|}
$22.3 \pm 0.5$ \\
$22.5 \pm 0.5$ \\
\end{tabular} & $\begin{array}{l}6.84 \pm 0.15 \\
6.90 \pm 0.15 \\
\end{array}$ \\
\hline 307 & 4.43 & & $30.0 \pm 0.5$ & $6.77 \pm 0.11$ \\
\hline 317 & 5.60 & & $37.2 \pm 1.5$ & $6.64 \pm 0.27$ \\
\hline 326 & 6.87 & \begin{tabular}{|l|}
$5.82^{\mathrm{a}}$ \\
$5.43^{\mathrm{b}}$ \\
$6.72^{\mathrm{c}}$ \\
$6.92^{\mathrm{d}}$ \\
$6.74^{\mathrm{c}}$
\end{tabular} & $47.8 \pm 2.0$ & $\begin{array}{l}6.96 \pm 0.29 \\
6.96 \pm 0.36\end{array}$ \\
\hline
\end{tabular}
(a) Ref. 27.
(b) Ref. 29.
(c) Ref. 30.
(d) Ref. 31 .
(e) Ref. 32 .
(f) Quoted errors are the statistical error of the fit.
(g) Quoted errors do not include the systematic uncertainty in the atom density.

at a couple of representative temperatures in Table 1). Thus, determination of cesium densities from the use of a vapor pressure formula suffers not only from problems associated with accurately measuring the liquid metal temperature, but also from possible errors in the vapor pressure

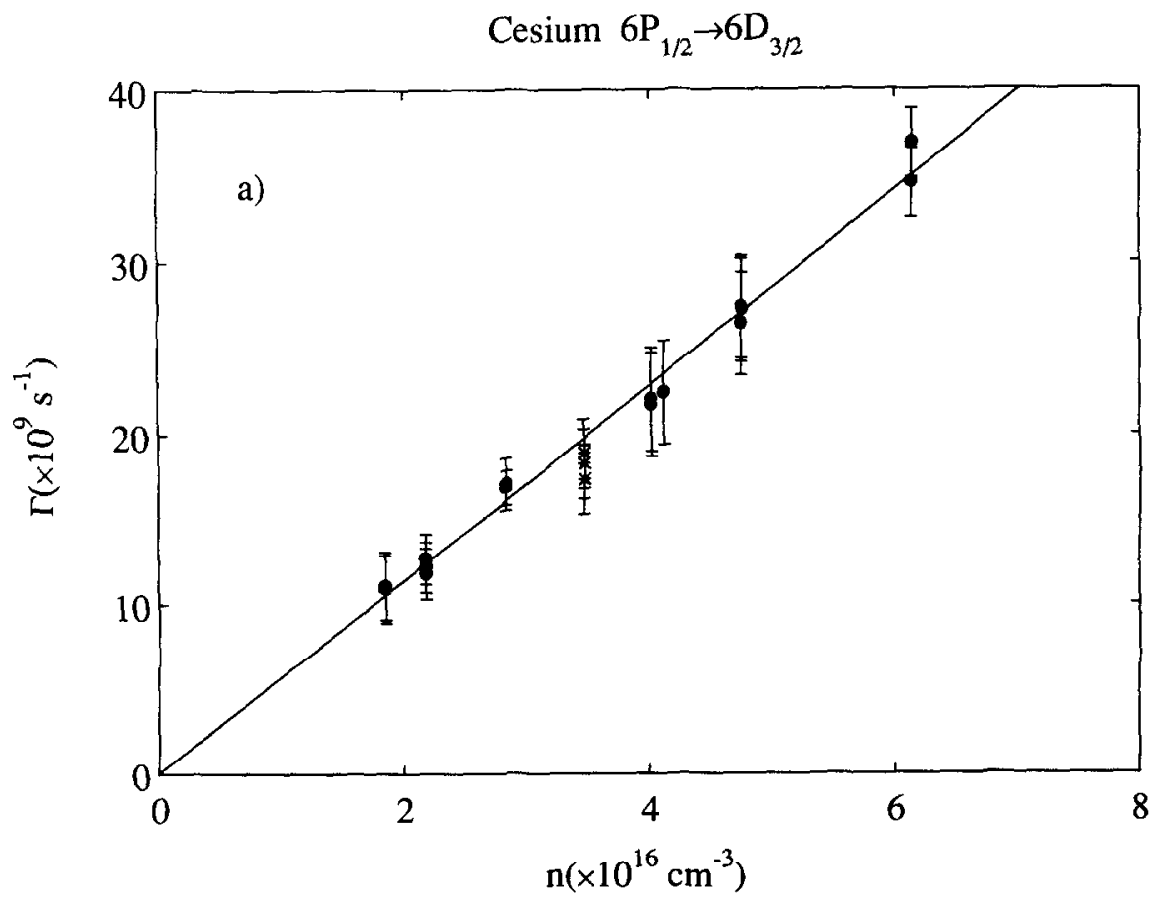

Fig. 5(a) (Legend opposite.) 
Cesium $6 \mathrm{P}_{3 / 2} \rightarrow 7 \mathrm{D}_{3 / 2}$

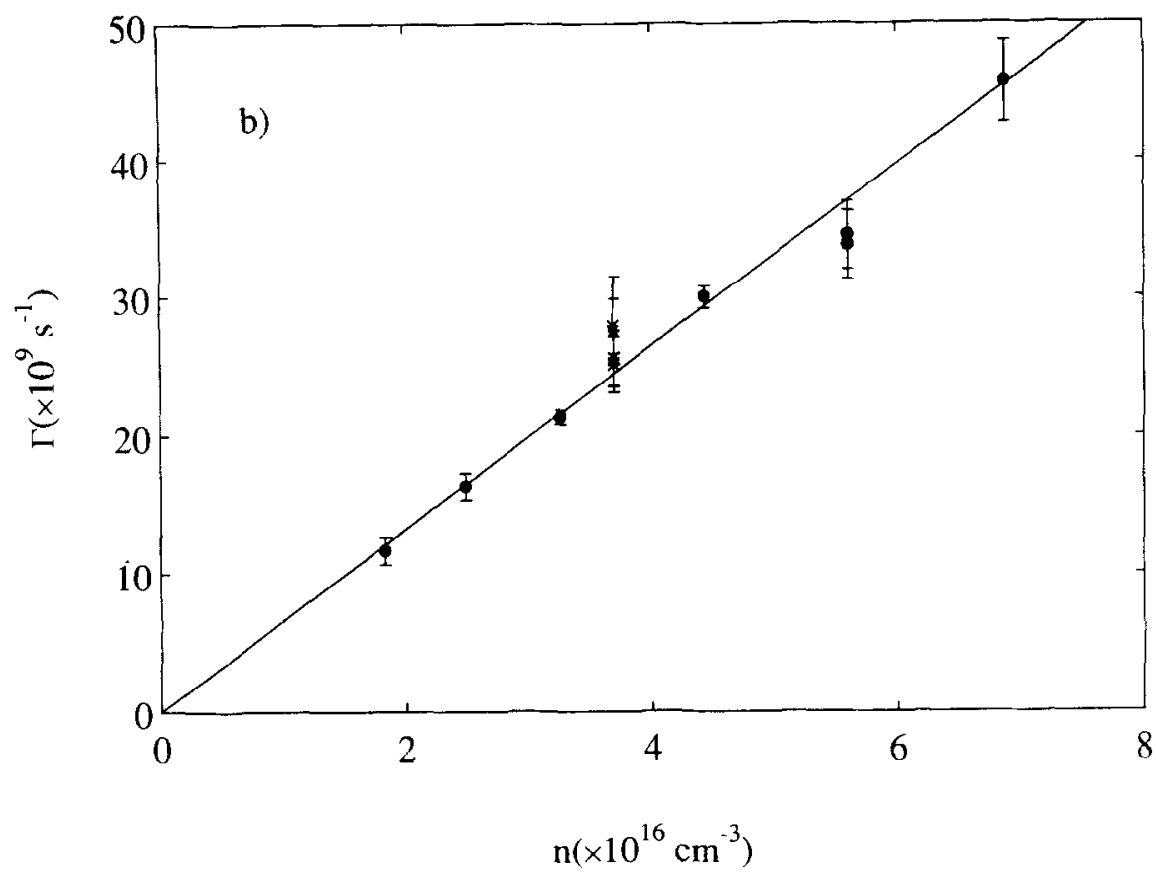

Cesium $6 \mathrm{P}_{3 / 2} \rightarrow 7 \mathrm{D}_{5 / 2}$

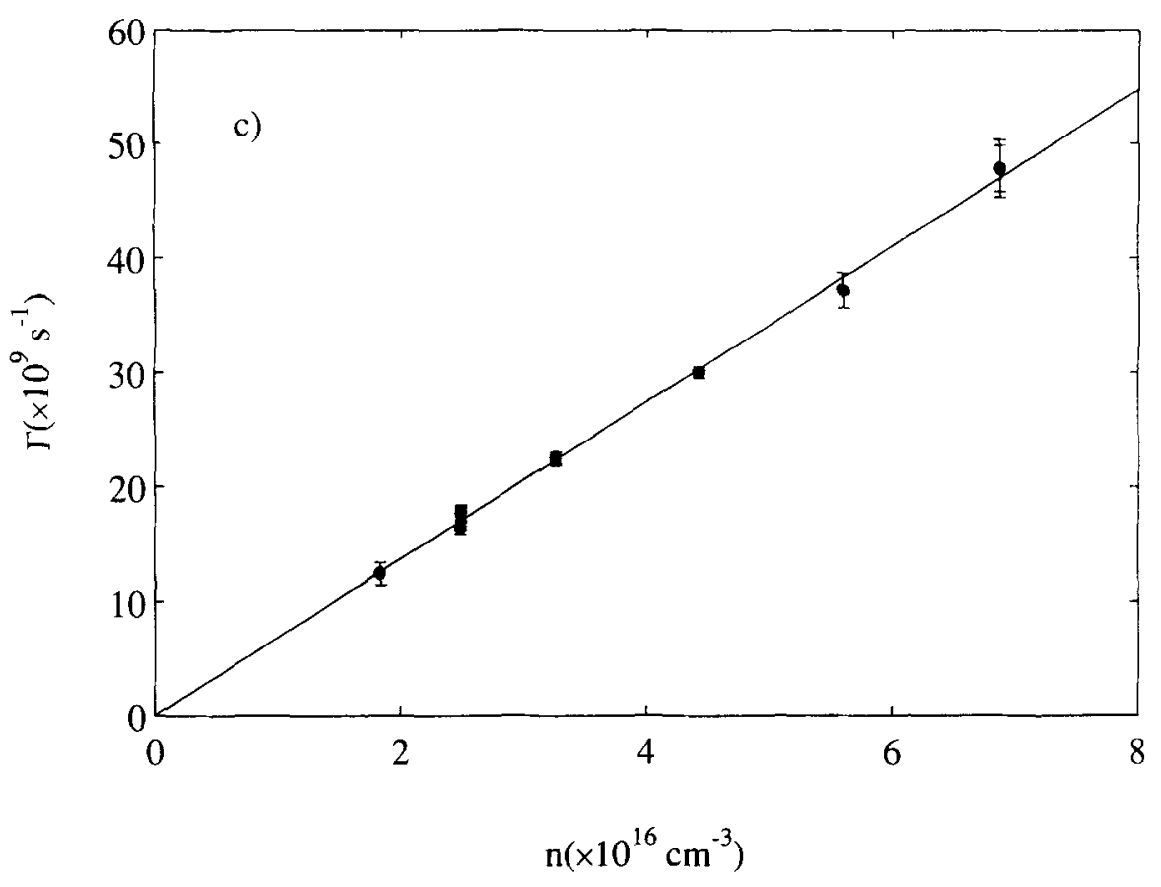

Figs. 5 (b) and (c)

Fig. 5. Plots of the measured Lorentzian broadening rates, $\Gamma_{b r}$, vs cesium atom density for (a) the $6^{2} P_{1 / 2} \rightarrow 6^{2} D_{3 / 2}$ transition, (b) the $6^{2} P_{3 / 2} \rightarrow 7^{2} D_{3 / 2}$ transition, and (c) the $6^{2} P_{3 / 2} \rightarrow 7^{2} D_{5 / 2}$ transition. Slopes of the least squares straight line fits to the data (solid lines) yield the resonance broadening rate coefficients $k_{\mathrm{br}}$. Data marked with * in Figs 5(a) and 5(b) were recorded on the same day without temperature cycling of the oven. 
formula itself, or in its extrapolation to the particular temperatures used in the experiment.

Nevertheless, we take the cesium density from the Nesmeyanov vapor pressure formula (which seems to be a good compromise among the more recent compilations) using the measured cold point temperature of the cell. Plots of the Lorentzian width $\Gamma$ vs density for the three transitions studied in this work are shown in Fig. 5. Least-squares straight line fits to Eq. (3) (using $\Gamma_{\text {nat }}$ as the fixed $y$-axis intercept) yield the experimental broadening rate coefficients:

$$
k_{\mathrm{br}}\left(D_{1}\right)=(5.7 \pm 1.0) \times 10^{-7} \mathrm{~cm}^{3} \mathrm{sec}^{-1}
$$

and

$$
k_{\mathrm{br}}\left(D_{2}\right)=(6.7 \perp 1.1) \times 10^{-7} \mathrm{~cm}^{3} \mathrm{sec}^{-1} .
$$

The major source of error here is due to use of the Nesmeyanov vapor pressure formula to obtain the cesium density. From line-center absorption measurements of the cesium $D_{1}$ line hyperfine components, we were able to verify the Nesmeyanov formula at room temperature to within $4.5 \%$, corresponding to a temperature discrepancy of $0.5^{\circ} \mathrm{C} .{ }^{24}$ Since this is equal to the temperature uncertainty, we believe the Nesmeyanov formula is fairly accurate in this low-temperature range. However, as stated above, the extrapolation of this temperature-density relation to the higher

Table 2. Experimental and theoretical values of the resonance broadening rate

\begin{tabular}{|c|c|c|c|}
\hline Reference & $k_{b r}\left(D_{2}\right) \quad\left(10^{-7} \mathrm{~cm}^{3} \mathrm{~s}^{-1}\right)$ & $k_{b r}\left(D_{1}\right),\left(10^{-7} \mathrm{~cm}^{3} \mathrm{~s}^{-1}\right)$ & $k_{b r}\left(D_{2}\right) / k_{b r}\left(D_{1}\right)$ \\
\hline \multicolumn{4}{|c|}{ Experimental } \\
\hline This Work & $\begin{array}{l}6.61 \pm 1.09 \\
6.84 \pm 1.09 b\end{array}$ & $5.69 \pm 0.98$ & $\begin{array}{l}1.18 \\
1.37^{\mathrm{c}} \\
1.48^{\mathrm{d}}\end{array}$ \\
\hline Gregory & $\begin{array}{l}9.11 \\
(7.80) \\
\end{array}$ & $\begin{array}{l}5.28 \\
(4.51)\end{array}$ & 1.73 \\
\hline Chen and Phelps ${ }^{5}$ & $\begin{array}{l}9.49 \pm 0.35 \\
(8.12 \pm 0.30)\end{array}$ & $\begin{array}{l}5.40 \pm 0.18 \\
(4.62 \pm 0.15)\end{array}$ & 1.76 \\
\hline Niemax and Pichler & $11.0 \pm 1.0$ & $8.2 \pm 0.5$ & 1.34 \\
\hline Niemax et. al. ${ }^{7}$ & $\begin{array}{l}7.9 \pm 2.4 \\
(6.8 \pm 2.1)\end{array}$ & $\begin{array}{l}5.3 \pm 1.6 \\
(4.5 \pm 1.4)\end{array}$ & 1.49 \\
\hline Vuletic et. al. ${ }^{13}$ & & $\begin{array}{l}4.7 \pm 0.7 \\
(4.0 \pm 0.6)\end{array}$ & \\
\hline Akul'shin et. al. ${ }^{14}$ & $\begin{array}{l}7.2 \pm 1.4 \\
(6.2 \pm 1.2) \\
\end{array}$ & & \\
\hline \multicolumn{4}{|c|}{ Theoretical } \\
\hline Carrington et. al. ${ }^{16}$ & 7.52 & 4.73 & 1.59 \\
\hline Ali and Griem ${ }^{17}$ & 6.94 & 5.03 & 1.38 \\
\hline Reck et. al. ${ }^{18}$ & 8.21 & 5.61 & 1.46 \\
\hline Movre and Pichler ${ }^{33}$ & 8.46 & 6.07 & 1.39 \\
\hline
\end{tabular}
coefficients $k_{\mathrm{hr}}$ for the cesium $D_{1}$ and $D_{2}$ transitions, and their ratio.

(a) Measured on the $6^{2} P_{3 / 2} \rightarrow 7^{2} D_{3 / 2}$ transition.

(b) Measured on the $6^{2} P_{3 / 2} \rightarrow 7^{2} D_{5 / 2}$ transition.

(c) Ratio measured from a single set of measurements [marked by * in Figs 5(a) and 5(b)] recorded on the same day without temperature cycling of the oven.

(d) Ratio determined from white light absorption equivalent widths measured between $T=203$ and $253^{\circ} \mathrm{C}$. 
temperatures of the present experiment introduces additional uncertainty. Nevertheless, because of the care taken in ensuring that the temperature of the coldest point in the cell was measured, we believe a realistic uncertainty in the density is $15 \%$. Other minor sources of error are due to statistical uncertainties in the broadening rates from the fitted line widths $(\sim 2 \%)$, and neglect of the van der Waals broadening and quadrupole-quadrupole broadening in the $6^{2} S_{1 / 2}+6^{2} S_{1 / 2}$ and $6^{2} S_{1 / 2}+n^{2} D_{J}$ manifolds, respectively $(\sim 0.1 \%)$.

Table 2 shows self-broadening rate coefficient values, for both the $D_{1}$ and $D_{2}$ lines, obtained from previous experiments. In Refs. 6 and 7 , measured $C_{3}$ coefficients are reported. We have converted these to $k_{\mathrm{br}}$ values by use of the purely formal expression $k_{\mathrm{br}}=\frac{4 \pi^{2}}{3} C_{3} \cdot{ }^{33}$ The relatively high values of Ref. 6 were attributed in Ref. 7 to a systematic error in the temperature determination. In the line wing studies of Gregory, ${ }^{4}$ Chen and Phelps, ${ }^{5}$ and Niemax et al, ${ }^{7}$ as well as in the selective reflection measurements of Vuletic et a ${ }^{13}$ and Akul'shin et al, ${ }^{14}$ densities were taken from the Taylor and Langmuir vapor pressure formula. Since these densities are systematically $\sim 15 \%$ smaller than the Nesmeyanov values in the relevant temperature range, we have also presented broadening rates from these references which were re-evaluated using Nesmeyanov densities for comparison to the present work. These adjusted values are given in Table 2 in parentheses. While our $D_{2}$ value is in the middle of those from previous measurements, our $D_{1}$ value is clearly at the high end, although it can be seen that all experimental values (with the exception of Ref. 6) agree to within uncertainties. Also in Table 2 we list theoretical calculations of the broadening coefficients from Carrington, Stacey, and Cooper, ${ }^{16}$ Ali and Griem, ${ }^{17}$ Reck et al, ${ }^{18}$ and Movre and Pichler ${ }^{34}$ Here it can be seen that our measured $D_{2}$ broadening rate coefficient is somewhat smaller than the theoretical values, while our $D_{1}$ coefficient is larger. In general, our values are in best agreement with the theoretical results of Ali and Griem, ${ }^{17}$ although our results also agree to just within uncertainties with the Carrington, Stacey, and Cooper ${ }^{16}$ results as well.

The ratio of our two measured rate coefficients $\left[k_{\mathrm{br}}\left(D_{1}\right) / k_{\mathrm{br}}\left(D_{2}\right)=1.18 \pm 15 \%\right]$ differs significantly from those obtained in previous measurements and theoretical calculations. This ratio should be relatively insensitive to systematic errors such as uncertainty in the cesium density. However, due to availability of equipment, our $D_{1}$ and $D_{2}$ broadening measurements were separated in time by several months (although the same cell and oven were used). Thus we decided to carry out one final set of measurements, at $T \sim 298^{\circ} \mathrm{C}$, where both the $D_{1}$ and $D_{2}$ broadening rates could be obtained under identical conditions, including identical cesium atom density. The results from this set of measurements agreed with our previous data within error bars, and these values are included in the plots shown in Fig. 5. However, the broadening rate ratio obtained from this final set of measurements $\left[k_{\mathrm{br}}\left(D_{1}\right) / k_{\mathrm{br}}\left(D_{2}\right)=1.37\right]$ is slightly larger than the ratio of our best values for the $D_{1}$ and $D_{2}$ broadening rate coefficients reported here, but still smaller than most of the previous experimental and theoretical ratios listed in Table 2. Finally, one additional experimental value for this ratio can be obtained from the ratio of absorption equivalent widths of the resonance lines in the regime where the equivalent width is dominated by self-broadening. In this limit it can be shown that the equivalent width $W_{i}$ is given by

$$
W_{i}=\frac{\lambda_{0}^{2}}{c}\left(\frac{\lambda_{0}^{2}}{8 \pi^{2}} \frac{g_{2}}{g_{1}} n^{2} \Gamma_{\text {nat }} L k_{\mathrm{br}}\right)^{1 / 2}
$$

where $g_{2}$ and $g_{1}$ represent the upper and lower state statistical weights, and $L$ is the absorption path length through the cell. Thus we find for the cesium $D_{1}$ and $D_{2}$ lines $W_{i}\left(D_{2}\right) / W_{\lambda}\left(D_{1}\right)=$ $1.30\left[k_{\mathrm{br}}\left(D_{2}\right) / k_{\mathrm{br}}\left(D_{1}\right)\right]^{1 / 2}{ }^{22.35}$ From a series of white light absorption scans at different temperatures in the range $203-253^{\circ} \mathrm{C}$, we have found $\left[k_{\mathrm{br}}\left(D_{2}\right) / k_{\mathrm{br}}\left(D_{1}\right)=1.48\right]$. However, at these temperatures, the equivalent widths range from 2.7 to $22 \AA$. Thus these measurements probe regions of the lineshape that are far outside the impact regime and well into the quasi-static region. It appears to us that the ratio $k_{\mathrm{br}}\left(D_{2}\right) / k_{\mathrm{br}}\left(D_{1}\right)$ is smaller in the line core than in the quasi-static wings by perhaps $10-20 \%$. In terms of theory, Reck et $\mathrm{al}^{18}$ and Movre and Pichler $^{34}$ are both quasi-static theories which should be valid in the line wings, while Carrington et $\mathrm{al}^{16}$ and Ali and Griem ${ }^{17}$ are impact regime theories. Carrington et $a^{16}$ mention that their value for $k_{\mathrm{br}}\left(D_{1}\right)$ is probably underestimated because mixing between singlet and triplet states of the emitter/perturber system does not appear in their theory, but can occur through terms of higher order than dipole-dipole. Thus they state that their value for $k_{\mathrm{br}}\left(D_{2}\right) / k_{\mathrm{br}}\left(D_{1}\right)$ is an upper limit. 


\section{CONCLUSIONS}

The present measurements of the self-broadening rate coefficients of the cesium resonance lines $\left[k_{\mathrm{br}}\left(D_{1}\right)=(5.7 \pm 1.0) \times 10^{-7} \mathrm{~cm}^{3} \mathrm{sec}^{-1}\right.$ and $\left.k_{\mathrm{br}}\left(D_{2}\right)=(6.7 \pm 1.1) \times 10^{-7} \mathrm{~cm}^{3} \mathrm{sec}^{-1}\right]$ are consistent with previous experimental and theoretical studies, although the ratio $k_{\mathrm{br}}\left(D_{2}\right) / k_{\mathrm{br}}\left(D_{1}\right)$ is somewhat smaller than predicted by theory or measured in line wing studies. These results also verify, to within experimental uncertainties, that the selective reflection method yields accurate results for line-broadening rate coefficients in the impact regime.

Acknowledgements-The authors would like to thank Drs. Alan Streater and A. Peet Hickman for many valuable discussions on this topic. We would like to acknowledge financial support for this work from the National Science Foundation under grant PHY-9119498.

\section{REFERENCES}

1. D. S. Hughes and P. E. Lloyd, Phys. Rev. 52, 1215 (1937).

2. S.-Y. Ch'en, Phys. Rev. 58, 884 (1940).

3. K. Watanabe, Phys. Rev. 59, 151 (1941).

4. C. Gregory, Phys. Rev. 61, 465 (1942).

5. C. L. Chen and A. V. Phelps, Phys. Rev. 173, 62 (1968).

6. K. Niemax and G. Pichler, J. Phys. B 7, 2355 (1974); 8, 179 (1975).

7. K. Niemax, M. Movre, and G. Pichler, J. Phys. B 12, 3503 (1979).

8. K. Niemax and G. Pichler, J. Phys. B 8, 179 (1975).

9. J. M. Vaughan, Proc. R. Soc. A 295, 164 (1966).

10. H. G. Kuhn and E. L. Lewis, Proc. R. Soc. $A$ 299, 423 (1967).

11. D. N. Stacey and R. C. Thompson, Acta Phys. Pol. A54, 833 (1978).

12. E. L. Lewis, M. M. Rebbeck, and J. M. Vaughan, J. Phys. B 4, 741 (1971).

13. V. Vuletić, V. A. Sautenkov, C. Zimmermann, and T. W. Hänsch, Opt. Commun. 99, 185 (1993).

14. A. M. Akul'shin, V. L. Velichanskiř, A. S. Zibrov, V. V. Nikitin, V. V. Sautenkov, E. K. Yurkin, and N. V. Senkov, JETP Lett. 36, 303 (1982).

15. J. Huennekens and A. Gallagher, Phys. Rev. A 27, 1851 (1983).

16. C. G. Carrington, D. N. Stacey, and J. Cooper, J. Phys. B 6, 417 (1973).

17. A. W. Ali and H. R. Griem, Phys. Rev. 140, A1044 (1965).

18. G. P. Reck, H. Takebe, and C. A. Mead, Phys. Rev. 137, A683 (1965).

19. E. L. Lewis, Phys. Rep. 58, 1 (1980).

20. T. Holstein, Phys. Rev. 79, 744 (1950).

21. E. Arimondo, M. Inguscio, and P. Violino, Rev. Mod. Phys. 49, 31 (1977).

22. A. Corney, Atomic and Lascr Spectroscopy, Clarendon, Oxford (1977).

23. B. Bussery and M. Aubert-Frécon, J. Mol. Spectrosc. 115, 169 (1986).

24. Z. J. Jabbour, J. Huennekens, M. Allegrini, S. Milošević, and F. de Tomasi, to be published.

25. D. G. Hummer, Mem. R. Astr. Soc. 70, 1 (1965).

26. B. Warner, Mon. Not. R. Astr. Soc. 139, 115 (1968).

27. J. B. Taylor and I. Langmuir, Phys. Rev. 51, 753 (1937).

28. A. N. Nesmeyanov, Vapor Pressure of the Elements, Academic, New York (1963).

29. K. K. Kelley, U.S. Bur. Mines, Bull. 383 (1935).

30. J. Bohdansky and H. E. J. Schins, J. Phys. Chem. 71, 215 (1967).

31. R. E. Honig and D. A. Kramer, RCA Rev. 30, 286 (1969).

32. C. B. Alcock, V. P. Itkin, and M. K. Horrigan, Can. Met. Quart. 23, 309 (1984).

33. J. P. Huennekens, Ph.D. thesis, University of Colorado (1982, unpublished).

34. M. Movre and G. Pichler, J. Phys. B 10, 2631 (1977); 13, 697 (1980).

35. A. C. G. Mitchell and M. W. Zemansky, Resonance Radiation and Excited Atoms, Cambridge University Press, Cambridge (1934). 